\title{
GANGA OPERATIVE SPINE COURSE
}

\section{Bucks fusion}

Published online: 4 February 2010

(C) Springer-Verlag 2010

\section{S. Rajasekaran MD, Vijay Kamath MD and Ashwin Avadhani MD}

\section{Keywords}

Direct pars repair $\cdot$ Bucks $\cdot$ Spondylolysis

\section{Introduction}

There are two major types of surgical procedures for spondylolysis, direct repair for a defect of the pars interarticularis and posterolateral fusion. Pars repair preserves segmental motion and is a less aggressive approach than lumbosacral fusion. Pars repair is thus preferred for patients with a painful spondylolysis without a degenerative caudal disc or an associated slip.

The pain generator in a patient with spondyloysis may be the pars defect, degenerated caudal disc, or of an unrelated pathology. Hence it is imperative that the pars defect be identified as the pain generator prior to undertaking this procedure. A diagnostic pars block is performed by infiltration of $0.2-0.3 \mathrm{ml}$ of $2 \%$ lidocaine into each pars defect and observing for relief of lumbago. A positive test (relief of lumbago) is an indication of the lysis as the pain source.

Electronic supplementary material The online version of this article (doi:10.1007/s00586-010-1308-y) contains supplementary material, which is available to authorized users.

\section{Case description}

The patient was a 16-year-old male with severe low back pain of 10 months duration which had not improved with conservative treatment. Lumbar movements especially extension were painfully restricted. He had normal neurology. Radiographs showed an associated bifid L5 and S1, with sclerosis of the pars bilaterally. The L5-S1 disc height was normal and there was no listhesis. CT scan showed a pars defect bilaterally with sclerosis of the margins. MRI showed a normal L5-S1 disc with no evidence of degeneration.

\section{Surgical procedure}

The patient was placed prone on bolsters on a radiolucent table. The normal lordosis of the lumbar spine was maintained.

The L5 lamina, including the pars interarticularis defect was exposed through a midline posterior incision. The affected level was easily identified by the abnormal mobility of its posterior arch. The fibrous tissue in and around the defect was removed with a curette. Care was taken not to remove the sclerotic bone excessively at the pseudarthrosis to avoid a large bone defect. If nerve-root decompression is indicated, the bone spurs overlying the affected nerve root can be removed with a Kerrison's rongeur. An entry point was made with the burr at the inferior edge of the lamina where it merges with the facet joint. Under flurocopic visualization, a standard 3.2-mm drill was used to drill the lower pars, across the defect and into the pedicle. It is preferable to penetrate the superior wall of the pedicle so that the screw can obtain a secure fixation. The length of the screw was assessed with a depth gauge.The bone around the pseudoarthrosis and the 
adjacent portions of the lamina are freshened using a burr. Bone chips from the ilium were packed into the defect, and a cortical screw introduced and tightened. Cancellous bone were additionally placed as an onlay graft over the pseudoarthrosis site.

In bilateral lysis with frank pseudoarthrosis it is important to insert the screws on both sides to engage the pedicles and then tighten simultaneously. Tightening the screw fully on one side will lead to a rotation of the rattler and lead to difficulties in the insertion of the second screw.

\section{Post-op procedure}

The radiograph is assessed for the screw position and trajectory and on follow-up visits to assess fusion. This is better observed in oblique views of the spine. Postoperatively, the patients are allowed to ambulate as soon as pain allows. A lumbosacral hard corset is worn till radiographs show fusion. Unrestricted activity is then allowed.

\section{Discussion and conclusion}

The indication for direct repair is symptomatic unilateral or bilateral spondylolysis without spondylolisthesis. Bucks technique has the advantage of providing direct compression across the defect and good results have been reported with healing rates ranging from 82 to $100 \%$. The ideal patient is 30 years of age or younger who is a non smoker. On radiographs the pars defect should be less than $3 \mathrm{~mm}$ and the disc at the level should be normal. Contraindications include a negative pars block implying that the spondylolysis is not the pain generator, Grade-II or greater spondylolisthesis and degeneration of the disc, and degenerative osteoarthrosis of the facet joint.

Disadvantages of this procedure are technical difficulty of accurate screw placement, that it cannot be used if the lamina is dysplastic and it may decrease the area available for bone graft [1-4].

\section{References}

1. Buck JE (1970) Direct repair of the defect in spondylolisthesis. Preliminary report. J Bone Joint Surg Br 52:432-437

2. van der Werf GJ, Tonino AJ, Zeegers WS (1985) Direct repair of lumbar spondylolysis. Acta Orthop Scand 56:378-379

3. Roca J, Moretta D, Fuster S, Roca A (1989) Direct repair of spondylolysis. Clin Orthop Relat Res 246:86-91

4. Deguchi M, Rapoff AJ, Zdeblick TA (1999) Biomechanical comparison of spondylolysis fixation techniques. Spine 24:328-333 